\title{
Lagging strand maturation factor Dna2 is a component of the replication checkpoint initiation machinery
}

\author{
Sandeep Kumar and Peter M. Burgers ${ }^{1}$ \\ Department of Biochemistry and Molecular Biophysics, Washington University School of Medicine, St. Louis, Missouri, USA 63110
}

\begin{abstract}
Initiation of the DNA replication checkpoint in yeast is mainly mediated by Mec1 protein kinase, the ortholog of human ATR, while its homolog Tel1, the ortholog of human ATM, has a minor replication checkpoint function. Checkpoint initiation requires stimulation of Mec1 kinase activity by specific activators. Saccharomyces cerevisiae Dna2, a nuclease-helicase that is essential for Okazaki fragment maturation, is employed specifically during $S$ phase to stimulate Mec1 kinase and initiate the replication checkpoint. Mutations (W128A and Y130A) in the unstructured $\mathbf{N}$ terminus of Dna2 abrogate its checkpoint function in vitro and in vivo. Dna2 shows partial redundancy for the replication checkpoint with checkpoint initiators 9-1-1 (S. cerevisiae Ddc1-Mec3-Rad17 and human Rad9-Rad1-Hus1) and Dpb11, the ortholog of human TopBP1. A triple mutant that eliminates the checkpoint functions of all three initiators abrogates the Mec1-dependent checkpoint.
\end{abstract}

[Keywords: DNA replication; cell cycle checkpoint; 9-1-1; ATR; ATM]

Supplemental material is available for this article.

Received August 30, 2012; revised version accepted December 26, 2012.

DNA damage resulting from internal or external insult constantly challenges cellular genome integrity. Analogous challenges are presented during DNA replication due to the presence of structural blocks or to replisome dysfunction. Eukaryotes have evolved several checkpoints that ensure an arrest of the cell cycle in order to provide an appropriate time frame for DNA repair or the completion of genome duplication (Hartwell and Weinert 1989). Checkpoints are initiated by the activation of two large protein kinases that belong to the phosphatidylinositol 3-kinase-related protein kinase (PIKK) family. Classically, checkpoints are grouped into two separate pathways: one that responds to dsDNA breaks, which activates Tell kinase (human ATM) (Morrow et al. 1995; Bakkenist and Kastan 2003), and one that responds to stretches of ssDNA coated with RPA (ssDNA-binding protein), which activates Mecl kinase (human ATR) (Sanchez et al. 1996; Zou and Elledge 2003). However, some redundancy exists for Mec1 and Tell checkpoint function. Of particular importance for this study, we note that stalled replication forks in yeast signal with partial redundancy to both Mec1 and Tel1 kinase, although Mec1 is the predominant kinase in this pathway (Morrow et al. 1995; Mallory and Petes 2000), and dominant mutations in TEL1 can further

${ }^{1}$ Corresponding author

E-mail burgers@biochem.wustl.edu

Article published online ahead of print. Article and publication date are online at http://www.genesdev.org/cgi/doi/10.1101/gad.204750.112. enhance the phenotypic suppression of mec1s mutants (Baldo et al. 2008).

Mec1 is constitutively associated with Ddc2 (human ATRIP), which helps in its localization to ssDNA regions by interacting with RPA (Rouse and Jackson 2002; Zou and Elledge 2003). However, localization of Mec1-Ddc2 to sites of DNA damage is not sufficient to convert it into a catalytically active complex. Specific sensors transduce the DNA damage response signal to Mecl and stimulate its kinase activity. Two sensor proteins identified to date are the PCNA-like heterotrimeric checkpoint clamp 9-1-1 (Saccharomyces cerevisiae Ddc1-Mec3-Rad17 and human Rad9-Rad1-Hus1) (Majka et al. 2006a) and the replication initiation factor Dpb11 (human TopBP1) (Kumagai et al. 2006; Choi et al. 2007; Mordes et al. 2008; Navadgi-Patil et al. 2011). These factors stimulate Mec1/ATR to phosphorylate many downstream proteins, including the principal downstream effector kinase Rad53 (the functional homolog of human Chk1), which propagates the checkpoint pathway (Sanchez et al. 1996).

Previous studies by us and others have demonstrated that yeast 9-1-1 and Dpb11 perform critical checkpoint functions by stimulating the catalytic activity of Mec1 during the G1 and G2 phases of the cell cycle (Puddu et al. 2008; Navadgi-Patil and Burgers 2009; Pfander and Diffley 2011). The G1-phase DNA damage checkpoint is mediated by the Ddc1 subunit of 9-1-1, while the function of Dpb11 is dispensable during G1 (Navadgi-Patil and Burgers 2009; Pfander and Diffley 2011). Mutation of two conserved 
aromatic amino acids in Ddc1 (ddc1-WW352,544AA) abrogates Mec1 kinase stimulation in vitro and the G1 DNA damage checkpoint. On the other hand, a fully active G2/M DNA damage checkpoint requires the functions of both 9-1-1 and Dpb11. In S. cerevisiae, 9-1-1 functions in two pathways: (1) It directly activates Mec1, dependent on the two conserved aromatic amino acids in Ddc1 (W352 and W544), and (2) it recruits Dpb11 to Mec1 through conserved phosphorylation of T602. The triple ddc1-WWT352,544,602AAA mutant is completely defective for the G2/M checkpoint because 9-1-1 fails to both stimulate Mec1 kinase and recruit Dpb11. To date, direct Mec1/ATR kinase stimulation by 9-1-1 has only been demonstrated in $S$. cerevisiae. However, the recruitment function of Ddc1/Rad9 is conserved in Schizosaccharomyces pombe and in metazoan (Furuya et al. 2004; Delacroix et al. 2007; Lee et al. 2007). Fusion to PCNA of the activation domain of human TopBP1, the ortholog of Dpb11, bypasses the requirement of 9-1-1 for establishing the DNA damage checkpoint (Delacroix et al. 2007).

Furthermore, mutants lacking the Mec1 stimulatory activity of $D D C 1$ and $D P B 11$ that completely abrogated the G1 and G2/M DNA damage checkpoints still showed the presence of a robust replication and S-phase DNA damage checkpoint (Navadgi-Patil et al. 2011). Even after potential contributions by Tell were eliminated (this study), a replication checkpoint remained, suggesting the presence of another Mecl stimulatory factor that is specific for the $S$ phase of the cell cycle.

Here, we show that Dna2 activates Mec1 kinase activity both in vitro and in vivo. Dna2 has both ssDNA exonuclease and $5^{\prime}-3^{\prime}$ DNA helicase activities (Budd et al. 1995; Bae et al. 1998). It plays an important role in Okazaki fragment maturation (Ayyagari et al. 2003), DNA resection during dsDNA break repair (Zhu et al. 2008; Mimitou and Symington 2009), and mitochondrial DNA maintenance (Zheng et al. 2008; Duxin et al. 2009). The nuclease activity of Dna2 also aids in stabilizing stalled DNA replication forks from collapsing ( Hu et al. 2012). Thus, Dna2 is a major contributor to genomic stability. A domain analysis of Dna2 has ascribed critical genome stability functions to each of its three domains: the unstructured N-terminal domain (NTD), the nuclease domain, and the helicase domain (Fig. 1A; Bae et al. 2001). Here, we show that Mec1 activation by Dna2 does not require its nuclease or helicase activity, but the activity resides in the unstructured NTD. Mutation of two aromatic residues in the NTD (W128A and Y130A) abrogates its checkpoint function. Also, the three checkpoint initiators known to date (Dna2, Dpb11, and the Ddc1/Rad9 subunit of 9-1-1) employ a similar strategy to activate Mec1 kinase.

\section{Results}

Dna2 specifically stimulates kinase activity of Mec1 in vitro

Our analysis of Dpb11 and the Ddc1 subunit of 9-1-1, both of which stimulate Mecl kinase, showed that
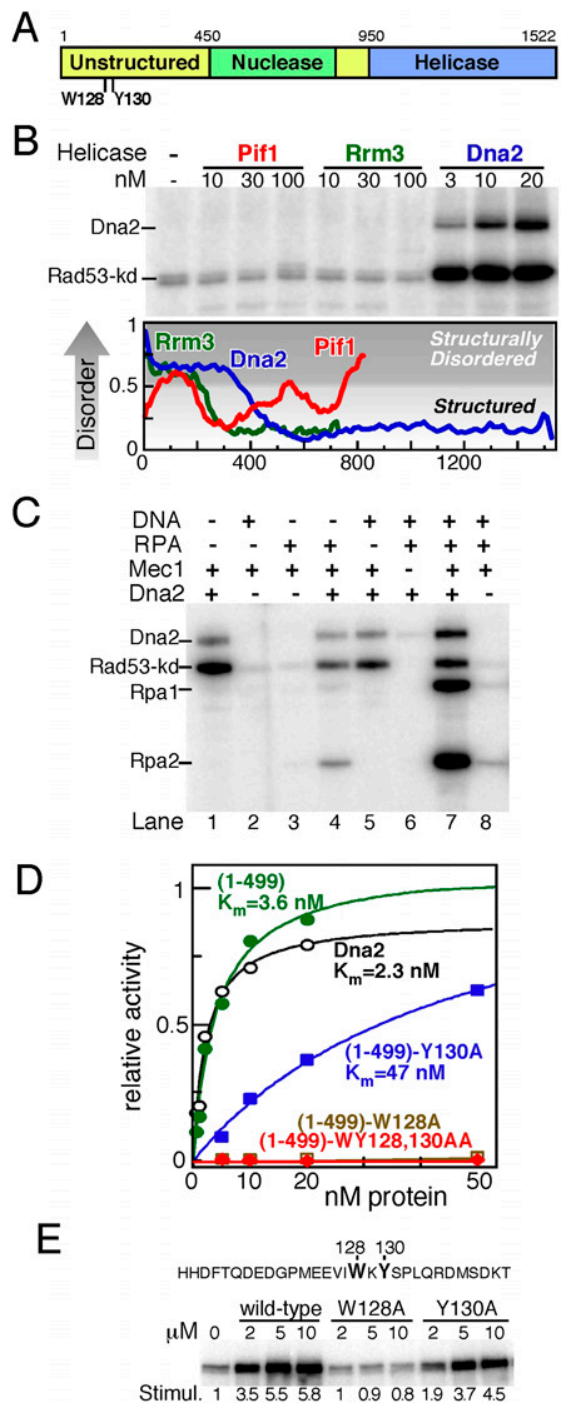

Figure 1. Dna2 activates Mec1 kinase in vitro. (A) Domain map of Dna2 depicting the unstructured domain and the nuclease and DNA helicase domains. Trp128 and Tyr130, required for Mec1 activation, are indicated. (B, top panel) Standard Mec1 kinase stimulation assays contain the indicated concentrations of Pif1, Rrm3, and Dna2 (see the Materials and Methods). (Bottom panel) Disorder prediction for Pif1, Rrm3, and Dna2. Disorder prediction programs IUPRED, PONDR, and PrDOS were used, and their output was averaged. Unstructured regions of proteins have a value $>0.5$. $(C)$ Standard Mec1 kinase stimulation assays were carried out with the indicated factors. (D) Michaelis-Menten analysis of the rate of Rad53-kd phosphorylation as a function of Dna2 or Dna2(1-499) wild-type or mutant concentration. (E) Sequence of a 30mer Dna2-derived peptide (amino acids 112-141) spanning the critical W128 and Y130. Mecl activation was carried out with the indicated concentrations of either wild-type or mutant peptide. Values at the bottom represent fold stimulation of Mecl activity.

stimulatory activity resides in an extended unstructured domain of these proteins (more than $\sim 40$ amino acids by phylogenetic analysis), containing at least two essential aromatic amino acids (Navadgi-Patil and Burgers 2009; Navadgi-Patil et al. 2011). Many replication/repair proteins 
contain such unstructured regions. Therefore, we tested purified proteins available in our laboratory for their ability to stimulate Mec1 activity, using Rad53-kd (Rad53-K227A; kinase-dead) as a physiological phosphorylation target. Out of 20 protein complexes surveyed (39 unique polypeptides), only Dna2 was shown to stimulate Mec1 kinase activity (Supplemental Fig. 1A,B).

Dna2 has an extended unstructured NTD (Fig. 1A). Many DNA helicases possess unstructured NTDs, as exemplified by the Pif1 and Rrm3 DNA helicases (Fig. 1B). However, even though both Pif1 and Rrm3 have several aromatic amino acids in their unstructured NTDs, they failed to stimulate Mecl protein kinase activity (Fig. 1B). Therefore, while an unstructured domain with aromatic amino acids appears to be a necessary determinant for Mec1 activation, it is not sufficient.

All kinase activity is Mec1-dependent (Fig. 1C, lane 6). As previously observed for Dpb11-mediated activation of Mec1 (Mordes et al. 2008; Navadgi-Patil et al. 2011), DNA is not required for Dna2 to stimulate Mec1 kinase (Fig. 1C, cf. lanes 1 and 5; Supplemental Fig. 1C). However, Mec1-mediated phosphorylation of the Rpal and Rpa2 subunits of RPA required that the RPA is bound to ssDNA (Fig. 1C, cf. lanes 4 and 7). Neither the nuclease nor helicase activities were required for Mec1 kinase stimulation (Supplemental Fig. 1D,E). This is consistent with the observation that the unstructured NTD (amino acids 1-499) of Dna2 is fully functional in Mec1 kinase stimulation (Fig. 1D). We noted the presence of several aromatic amino acids in the NTD, but only mutation to alanine of W128 and Y130 significantly affected the Mec1 stimulatory activity. The Y130A mutant reduced the apparent affinity of Dna2(1-499) for Mec1 by $\sim 15$-fold, whereas the W128A mutant showed very low kinase activation ( $\sim 2 \%$ of wild type) The double mutant dna2WY128,130AA, hereafter called dna2-WY-AA, was completely defective (Fig. 1D; see also Supplemental Fig. 1F). Consistent with these results, we found that a small Dna2-derived oligopeptide containing the Trp128-Tyr130 motif displayed Mec1 stimulatory activity, and this activity was reduced in the Y130A mutant peptide and abrogated in the W128A mutant peptide (Fig. 1E).

\section{The unstructured NTD of Dna2 can activate the kinase activity of Mec1 in vivo}

Next, we asked whether the Dna2 NTD could function as a $\mathrm{Mecl}$ activator in vivo by fusing this domain to an activation-defective 9-1-1 clamp (Fig. 2A). The 9-1-1 clamp is a heterotrimer of Ddc1, Rad17, and Mec3 (human Rad9, Hus1, and Rad1, respectively) (Parrilla-Castellar et al. 2004). While the entire complex and the clamp loader are essential for its checkpoint function, all Mec1 stimulatory activity resides in the $\mathrm{C}$ terminus of $\mathrm{Ddc1} /$ Rad9 (Navadgi-Patil and Burgers 2009). In fact, the artificial colocalization in S. cerevisiae of just Ddc1 with Mec1 causes gratuitous checkpoint activation (Bonilla et al. 2008). A DDC1 mutant [ddc1-(1-404 $\left.\left.{ }^{W A}\right)\right]$ that lacks its unstructured $\mathrm{C}$-terminal tail and carries an additional W352A mutation in the PCNA-like domain is defective
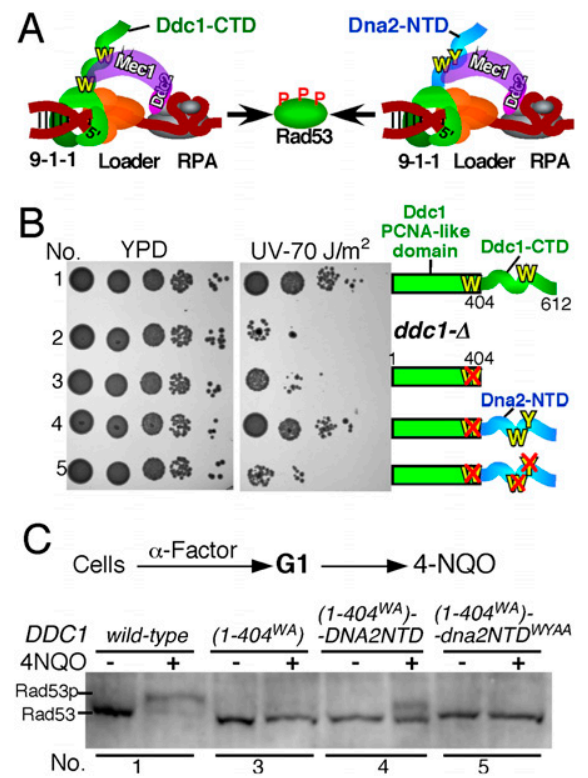

Figure 2. The Dna2 NTD is functional in vivo. (A) Cartoon diagram depicting activation of Mec1 by the 9-1-1 clamp, loaded onto DNA by the checkpoint clamp loader (Majka et al. 2006b). Activation is either through interaction between the Ddc1 C-terminal domain (CTD) (left) or with the Dna2 NTD fused to a tail-less form of Ddc1 (right). (B) Serial dilutions of a $d d c 1 \Delta$ strain (YLL244) transformed with a plasmid containing either wildtype DDC1, empty vector, $d d c 1-\left(1-404^{\mathrm{W} 352 \mathrm{~A}}\right), d d c 1-\left(1-404^{\mathrm{W} 352 \mathrm{~A}}\right)-$ DNA2(41-243), or $d d c 1-\left(1-404^{\mathrm{W} 352 \mathrm{~A}}\right)-d n a 2(41-243)^{\mathrm{WY}-\mathrm{AA}}$ were tested for sensitivity to $\mathrm{UV}_{254}$ irradiation $\left(70 \mathrm{~J} / \mathrm{m}^{2}\right)$. (C) Western analysis of Rad53 phosphorylation in cells arrested in G1 with $\alpha$ factor $(5 \mu \mathrm{g} / \mathrm{mL})$ and treated with 4-NQO $(2 \mu \mathrm{g} / \mathrm{mL})$ for $45 \mathrm{~min}$. The numbers below the figure refer to the same specific strain as used in $B$.

for the G1 and G2/M checkpoint (Navadgi-Patil and Burgers 2009) and shows profound sensitivity to UV (Fig. 2B, row 3). We fused the active region of the Dna2 NTD (amino acids $41-243$ ) to the C terminus of this mutant Ddc1 truncation, and this form of 9-1-1 was efficiently loaded onto DNA by the checkpoint clamp loader and stimulated Mec1 kinase-like wild-type 9-1-1 (Supplemental Fig. 2). The fusion gene also restored UV resistance of the ddc1 mutant (Fig. 2B, row 4). However, point mutations in the two aromatic amino acids that abrogated the stimulatory activity of Dna2 (Fig. 1D) also eliminated UV resistance (Fig. 2B, row 5). The observed UV resistance is likely a direct consequence of a functional checkpoint in cells containing the fusion gene. The G1 DNA damage checkpoint is defective in the DDC1 truncation mutant (Fig. 2C, No. 3), is restored to significant levels in cells with the fusion gene (Fig. 2C, No. 4), and is again inactivated when this fusion gene is mutated at the amino acids critical for Dna2's checkpoint activity (WY-AA) (Fig. 2C, No. 5). These data show that the NTD of Dna2 can function as a G1 checkpoint factor, but only because it is brought into conjunction with Mec1 onto damaged chromatin through loading of the 9-1-1 clamp. Dna2 itself does not function independently as a check- 
point protein in G1 or G2/M because (1) the stimulationdefective mutant dna2-WY-AA showed no defect in either the G1 or G2/M DNA damage checkpoint (Fig. $3 \mathrm{~A})$, and (2) deletion of any of the 9-1-1 genes shows a complete checkpoint defect in G1 and G2/M, excluding a significant compensatory function by Dna2 (Fig. 3A; Longhese et al. 1997; Kondo et al. 1999; Navadgi-Patil and Burgers 2009). We therefore investigated a potential checkpoint function for Dna2 during the $S$ phase of the cell cycle.

\section{Dna2 is a specific checkpoint activator for Mec1} during $S$ phase

The replication checkpoint is initiated in response to treatment of cells with hydroxyurea, an inhibitor of ribonucleotide reductase. The replication checkpoint is more complex than the G1 and G2 checkpoints because partial redundancies exist for the two apical kinases Mec1 and Tell (Morrow et al. 1995; Mallory and Petes 2000; Myung and Kolodner 2002). In addition, we anticipated that complete abrogation of the replication checkpoint might result in lethality, since the MEC1 deletion itself confers lethality. Lethality of mec1s is suppressed by deletion of $S M L 1$, a gene that negatively regulates ribonucleotide reductase (Zhao et al. 1998). Therefore all of our checkpoint studies were carried out in a sml1s background.

We used a set of isogenic strains that were all derived from heterozygous diploid strain PY301. The diploid was sporulated, and the desired mutations were identified in the spores by marker selection and PCR genotyping. Subsequently, the DNA2 mutations were introduced by a plasmid shuffle in which chromosomal dna2s cells containing DNA2 on a URA3 plasmid were transformed with a TRP1 plasmid containing either wild-type or mutant DNA2 or empty vector, and subsequently, the strains were plated on 5-fluoroorotic acid-containing medium in order to evict the complementing URA3-DNA2 plasmid. The resulting strains were immediately analyzed for growth and checkpoint phenotypes. This approach limits the prolonged propagation of poorly growing mutants and the associated generation of suppressors that occur readily due to the extremely high genome instability of fully checkpoint-defective yeast strains (Myung and Kolodner 2002). All strains were sml1s and either $d d c 1 \Delta$ and/or tel1s. Since the Mec1 stimulatory activity of Dpb11 is absolutely dependent on its interaction with the phosphorylated tail of Ddc1 (Furuya et al. 2004; Navadgi-Patil and Burgers 2009), using a $d d c 1 \Delta$ mutant ensured that checkpoint contributions by Dpb11 were also eliminated. In these mutant backgrounds, we probed whether the Mec1 stimulatory activity of Dna2 contributed to the replication checkpoint and the S-phase DNA damage checkpoint.

During checkpoint activation, Rad53 is initially phosphorylated by Mec1 and/or Tell kinase. Further propagation of the checkpoint and full hyperphosphorylation of Rad53 requires either the Rad9 or Mrcl mediator (Alcasabas et al. 2001; Osborn and Elledge 2003). Rad9 is the putative
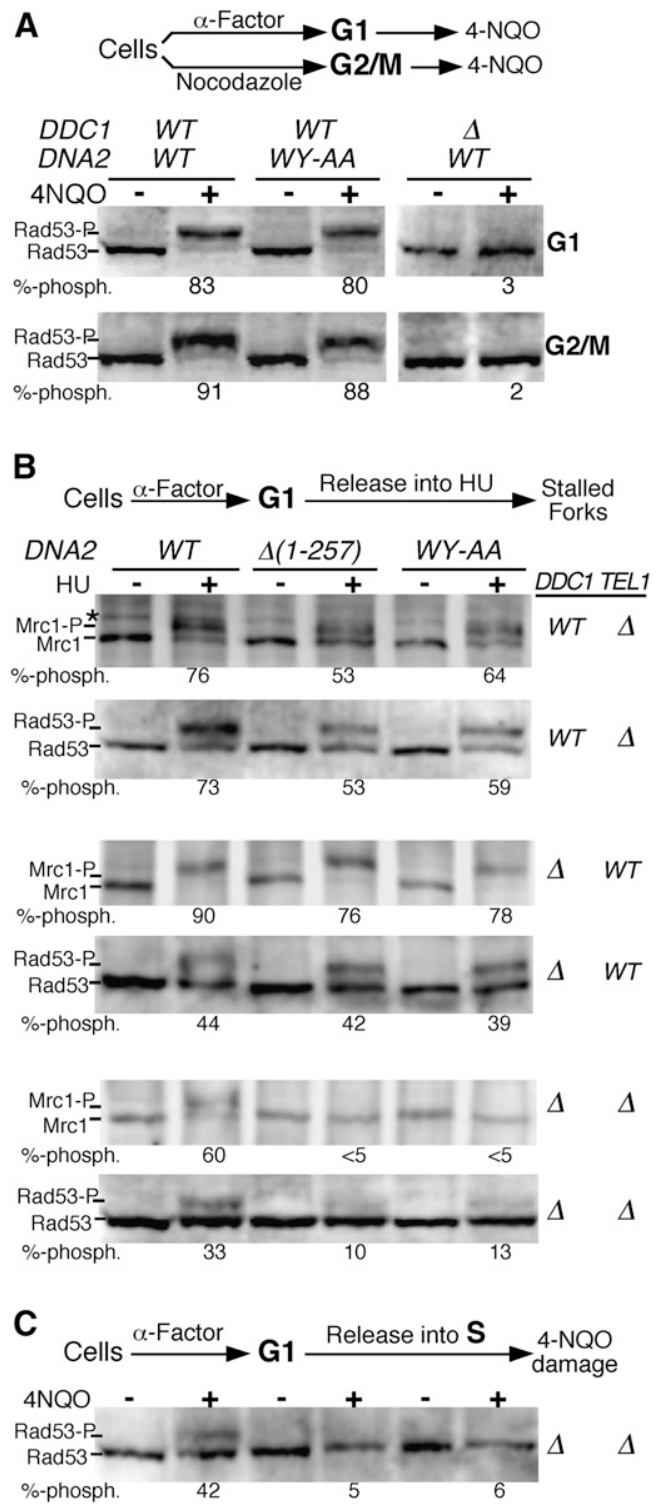

Figure 3. Dna 2 activates Mec 1 specifically during $S$ phase. $(A)$ Yeast strains PY288 and PY282, with the indicated genotypes, were arrested in G1 phase with $\alpha$ factor or in G2 phase with nocodazole $(20 \mu \mathrm{g} / \mathrm{mL}$; bottom half) and then treated with 4NQO for $30 \mathrm{~min}$. Western analysis of Rad53 phosphorylation was carried out as described in the Materials and Methods. Percentage of phosphorylated Rad53 is indicated. (B) Strains PY290, PY282, and PY286, with the indicated genotypes and with either wild-type DNA2 or the indicated dna2 mutants, were synchronized in G1 with $\alpha$ factor and released into fresh YPD with $200 \mathrm{mM}$ hydroxyurea for $90 \mathrm{~min}$. Cell extracts were probed with anti-Mrc1 or anti-Rad53 antibodies. (C) PY286 was synchronized as described above and released into fresh YPD with 4-NQO for 45 min. Analysis for Rad53 phosphorylation was as under $B .\left(^{*}\right)$ The appearance of a nonspecific band.

ortholog of human 53BP1 and primarily transduces the checkpoint in response to DNA damage, while Mrc1 is the ortholog of human claspin and responds to both damage and replication stress (for review, see Branzei and Foiani 
2009). The function of either mediator requires phosphorylation by Mec1 and/or Tell. Therefore, as phenotypic readouts of checkpoint activity, we measured the extent of hyperphosphorylation of Rad53, Mrc1, and Rad9 in response to replication fork stalling by hydroxyurea and in response to DNA damage caused by 4-nitroquinoline1-oxide (4-NQO), a UV-mimetic drug.

We determined the phenotypes of two DNA2 mutants: the dna2-WY-AA mutant that fails to stimulate Mecl in vitro (Fig. 1D) and that of a dna2- $\Delta(1-257)$ mutant that deletes the entire relevant region of the NTD (Chen et al. 2011). In all checkpoint assays, the phenotype of dna2$W Y-A A$ was comparable with that of $d n a 2-\Delta(1-257)$ (Fig. $3 \mathrm{~B}, \mathrm{C}$; Supplemental Figs. 3, 4). Furthermore, neither DNA2 mutation caused a detectable growth defect or checkpoint phenotype by itself; i.e., in an otherwise wildtype strain (Figs. 3A, 5A [below]; Supplemental Fig. 3).

A robust replication checkpoint in response to hydroxyurea treatment was observed in a wild-type cell (Supplemental Fig. 3A) and in a tel14 mutant (Fig. 3B). This is evident from the strong hyperphosphorylation of both Mrc1 and Rad53. Rad9 does not mediate the replication checkpoint, and in agreement with other studies (Alcasabas et al. 2001; Osborn and Elledge 2003), we did not observe significant Rad9 phosphorylation in either wild-type or mutant strains upon hydroxyurea treatment (Supplemental Fig. 3A). Introduction of the dna2-WY-AA or dna2-A(1-257) mutation into the tel1s strain significantly reduced the extent of both Mrcl and Rad53 phosphorylation (Fig. 3B). Similar results were obtained when we introduced the DNA2 mutations into the single $d d c 1 \Delta$ strain; a minor reduction in Mrc1 and Rad53 phosphorylation was observed in the double mutants (Fig. 3B).

Next, we examined the phenotype of DNA2 mutations in the $d d c 1 \Delta$ tel1 $\Delta$ double mutant. In a wild-type DNA2 background, the $d d c 1 \Delta$ tel1s double mutant still showed considerable Mrc1 phosphorylation in response to hydroxyurea treatment, although less robust than either the $d d c 1 \Delta$ or tel1s single mutant. However, strikingly, this phosphorylation signal was abrogated when, in addition, the Mec1 stimulatory function of DNA2 was mutated. Consistent with previous observations that Mrcl phosphorylation is required in order to transduce the replication checkpoint signal to Rad53 (Alcasabas et al. 2001), negligible Rad53 phosphorylation was also detected in the triple mutants after hydroxyurea treatment. Thus, the replication checkpoint is essentially eliminated in the triple mutants (Fig. 3B).

In order to measure the S-phase DNA damage checkpoint, cells were synchronized in G1 phase with $\alpha$ factor, then allowed to proceed into $S$ phase in fresh growth medium and treated with 4-NQO. Robust phosphorylation of both Rad9 and Mrc1 was observed in a wild-type and a tel1s strain. However, in the $d d c 1 \Delta$ mutant, Rad9 phosphorylation was undetectable, while that of Mrc1 was significantly reduced (Supplemental Fig. 3B). In the ddc1s tel1s double mutant, phosphorylation of both Rad9 and Mrc1 was below the detection limit (Supplemental Fig. 3B), but significant Rad53 phosphorylation was still observed (Fig. 3C). The residual S-phase DNA damage checkpoint activity remaining in the tel1s ddc1s double mutant was abrogated in the triple tel1s $d d c 1 \Delta$ dna2 mutants, as indicated by a complete lack of Rad53 phosphorylation upon 4-NQO treatment of S-phase cells (Fig. 3C).

\section{Mec1 kinase activation is required for growth}

During these studies, we noticed a very low viability $(<1 \%$ plating efficiency) of the tel1s ddc1s dna2 triple mutants (Fig. 4A; Supplemental Fig. 4). Mutants defective for both $M E C 1$ and TEL1 show poor viability and display a highly increased rate of genome instability compared with the single mutants (Myung and Kolodner 2002; Vernon et al. 2008). We determined that the poor viability of our triple mutants was a direct consequence of a defect in Mecl kinase activation. First, reduced viability was

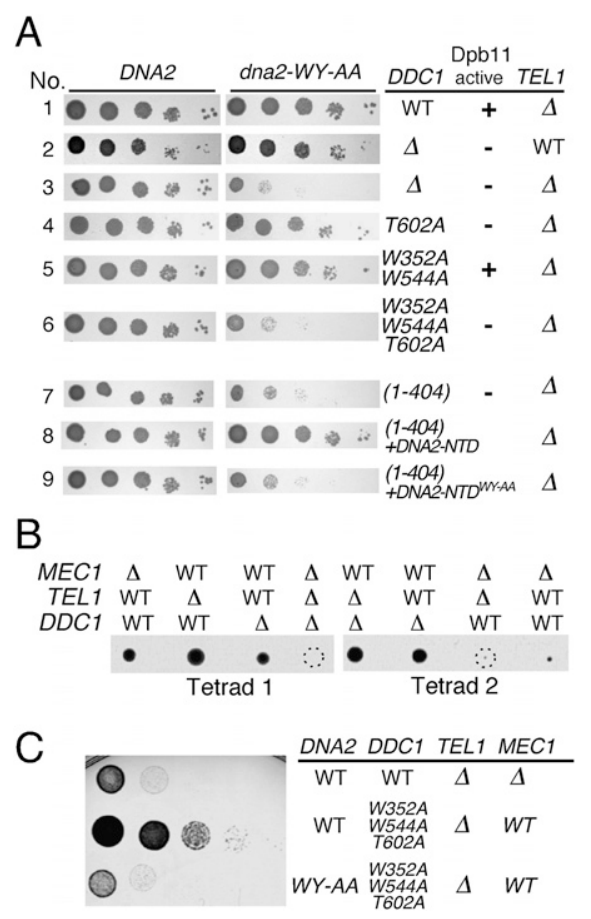

Figure 4. Cell viability and S-phase progression require minimal checkpoint kinase activity. (A) Tenfold serial dilutions of cells containing the indicated DDC1 and/or TEL1 mutation and either wild-type DNA2 (left) or dna2-WY-AA (right) were plated on 5 -fluoroorotic acid medium, which reveals the phenotype of mutant DNA2 by eviction of a wild-type DNA2-complementing plasmid (see the Supplemental Material). All strains are also sml1s. Complete plates are in Supplemental Figure 4. (B) Meiotic tetrads from a cross between PY305 (tel1s, sml1s) and PY310 (ddc1s mec1s sml1s) were dissected on YEPD plates, which were incubated for $5 \mathrm{~d}$ at room temperature. The relevant genotypes are indicated on top, and mec1s tel1s sml1s mutants are circled. $(C)$ Comparison of growth rate between a mec1s tel1s sml1s triple deletion strain (colony 3 from tetrad 2) and strain PY286 with the indicated $d d c 1$ allele and either wild-type DNA2 or dna2-WY-AA (see row 6 in $A$ ). See the Supplemental Material for further information and Supplemental Figure 4C for full plates with additional mutants. 
observed only in the triple mutant ddc1s tel1s dna2-WY$A A$, and restoring any of the three mutations to wild type restored robust growth (Fig. 4A, rows 1-3). Second, instead of the $d d c 1 \Delta$ mutant, we used individual $D D C 1$ point mutants that eliminate specific checkpoint functions. Stimulation of Mec1 by 9-1-1 is eliminated in a ddc1-WW352,544AA mutant, and participation of Dpb11 in checkpoint activation is eliminated in a $d d c 1-T 602 \mathrm{~A}$ mutant (Navadgi-Patil and Burgers 2009). Neither the ddc1-WW352,544AA mutant (Fig. 4A, row 5) nor the ddc1-T602A mutant (Fig. 4A, row 4) caused poor growth in a tel1s dna2-WY-AA background; however, the triple ddc1-WWT352,544,602AAA mutant (Fig. 4A, row 6), which eliminated stimulation by both Ddc1 and Dpb11, resulted in poor growth.

We carried out several other control studies to investigate the possibility of growth defects in the DNA2 mutants that would be unrelated to its checkpoint function. The essentiality of DNA2 stems from its function during Okazaki fragment maturation (for review, see Burgers 2009). Both the flap endonuclease FEN1 and the nuclease function of Dna2 process $5^{\prime}$ flaps generated during the process of Okazaki fragment maturation. Growth defects of conditional dna2 mutants are suppressed by overexpression of RAD27, which encodes FEN1 (Budd and Campbell 1997). However, RAD27 overexpression did not suppress the growth defect of our checkpoint-defective mutants, indicating that the growth phenotype of the dna2 mutants is checkpoint-specific (Supplemental Fig. 5A). Furthermore, our hypothesis predicts that any action that restores some checkpoint activity should also restore robust growth. Above, we showed that a chimeric ddc1-dna2 construct partially suppressed the checkpoint defects of $d d c 1\left(1-404^{W 352 A}\right)$ lacking its C-terminal tail (Fig. 2). This chimeric gene restored partial G1 checkpoint activity (Fig. 2C) and also partial S-phase DNA damage checkpoint activity (Supplemental Fig. 5B). The chimeric gene also suppressed the growth defect of the $d d c 1 \Delta$ tel1s $d n a 2-W Y-A A$ triple mutant, but suppression was lost when the critical aromatic amino acids in the Dna2 tail of the chimera were mutated (Fig. 2C, cf. rows 7 and 8,9). Finally, the dna2 mutants showed no growth defect in a mec1s sml1s $d d c 1 \Delta$ strain that was wild type for TEL1 (Supplemental Fig. 5C). From these data, we conclude that Mec1 needs to exert its kinase function, mediated by one of its three activator proteins (Ddc1, Dpb11, or Dna2), for robust growth in a tel1s mutant. Consistent with this conclusion, we found that a mec1s tel1s double mutant showed a growth defect comparable with that of the activation-

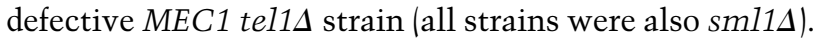
A tetrad analysis of a diploid strain that was heterozygous MEC1/mec1s and TEL1/tel1s but homozygous sml1s/ $s m l 1 \Delta$ showed poor or no spore viability of mec1s tel1s double mutants (Fig. 4B), and those double mutants that did grow were as defective for growth as the activationdefective MEC1 tel1A strain (Fig. 4C).

The poor growth that we observed in the multiple checkpoint-defective mutants is in part caused by their poor progression through $\mathrm{S}$ phase. A dramatic deterioration in S-phase progression was observed when tel1s ddc1s double mutants lost the Mec1 stimulatory function of Dna2 (Fig. 5A, panel 4). The response of these mutants to hydroxyurea also showed strong differences. Tel1 $\Delta$ ddc1 $\Delta$ double mutants progressed slowly but synchronously through $S$ phase in the presence of hydroxyurea, indicative of a functional checkpoint (Fig. 5B, panel 3). However, mutants that, in addition, have lost the Mec1 stimulatory function of Dna2 terminally accumulate in $S$ phase (Fig. 5B, panel 4). The mutant dna2 allele alone showed no cell cycle defect with or without hydroxyurea. This cell cycle analysis suggests that cells fail to properly complete DNA replication in checkpoint-defective mutants. Unreplicated chromosomes fail to migrate through agarose gels in a pulsed-field gel electrophoresis (PFGE) experiment (Hennessy et al. 1991). The migration defect of a ddc1s tel1s double mutant is strongly increased when this mutant also carries the dna2-WY-AA allele (Fig. 5C).

\section{Discussion}

The three Mec1 activators described in this study show a remarkable similarity of structure and mechanism of action. The activity of all three is localized to a region of the protein that is predicted to be disordered (Fig. 1B; Navadgi-Patil et al. 2011) The activity is anchored by the presence of two aromatic amino acids; however,

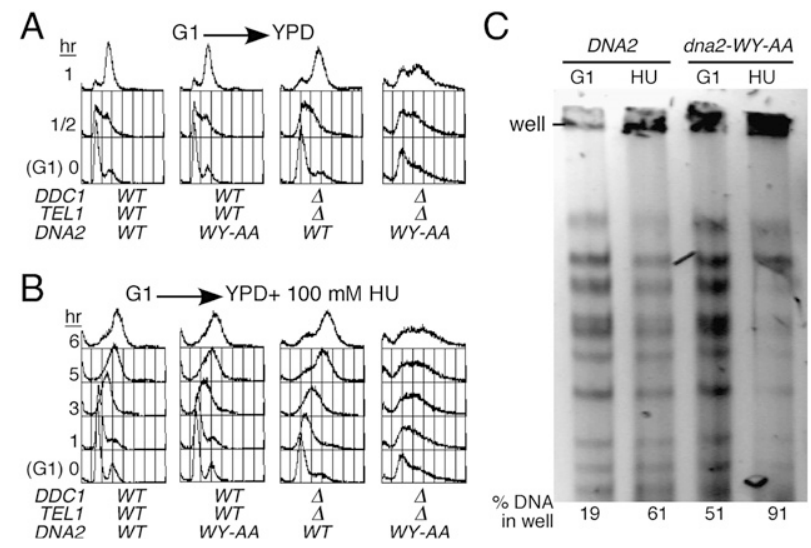

Figure 5. Checkpoint-defective mutant shows poor progression through S phase. FACS scans for strain PY288 (panels 1,2) and PY286 (panels 3,4). The relevant genotypes are indicated at the bottom of the profiles. Strains were synchronized in G1 with $\alpha$ factor and, at $t=0$, released into either fresh YPD $(A)$ or YPD with $100 \mathrm{mM}$ hydroxyurea $(B)$. (C) Strain PY286 with either $D N A 2$ or dna2-WW-AA was synchronized in G1 with $\alpha$ factor and released in YPD with or without $100 \mathrm{mM}$ hydroxyurea for $4 \mathrm{~h}$. Chromosomes was analyzed using PFGE (see the Supplemental Material). Values at the bottom represent the percentage of DNA remaining in the wells. Under normal growth conditions, only Chromosome XII fails to migrate into the gel (Carle and Olson 1985). Incompletely replicated chromosomes such as those that exist when cells are treated with hydroxyurea also tend to remain in the wells (Hennessy et al. 1991). The high percentage of DNA remaining in the well in the dna2-WY-AA mutant indicates that chromosomes fail to complete replication. 
surprisingly, in S. cerevisiae, these aromatics can be separated by as much as 191 amino acids in Ddc1 and as little as one in Dna2. In other organisms, a similar apparent lack of constraint exists. In the case of 9-1-1, the separation of the aromatics is reduced to 67 amino acids in human and 45 amino acids in $S$. pombe Rad9, the ortholog of Ddc1. This large variation in spacing makes predicting a region that will function as a Mec1/ATRactivating domain currently very difficult. Although all Dna2s, including vertebrate species, have an unstructured NTD containing several aromatic amino acids, predicting which ones will be involved in activation has not been possible, and experimental verification is necessary. It is quite evident, however, that there are definite constraints on the type of unstructured domain that can function as a Mecl/ATR activator. Other proteins with long unstructured regions, containing aromatic amino acids (e.g., Pif1 and Rrm3) (Fig. 1B), do not activate Mec1. In addition, our genetic analysis strongly suggests that the number of activators is limited to the three discovered so far: 9-1-1, Dpb11/TopBP1, and now Dna2. Elimination of the activation function of all three proteins leads to an essentially complete elimination of the DNA damage and replication checkpoints, provided a secondary pathway involving Tel1, the ortholog of human ATM, is also eliminated (Figs. 3, 6). Mec1 tel1 mutants also show telomere dysfunction (Myung and Kolodner 2002). The senescence phenotype of telomere dysfunction is generally expressed after prolonged cultivation of mutants (Lundblad and Szostak 1989). However, the immediate growth defects that we observed in our checkpoint-defective mutants suggest that some Mecl/Tell kinase activity is essential for normal mitotic cell growth (Figs. $4 \mathrm{~B}, 5 \mathrm{~A})$.

For each activator, we determined how elimination of its function by mutation affected phosphorylation of the mediator proteins $\mathrm{Mrcl}$ and Rad9 and of the effector

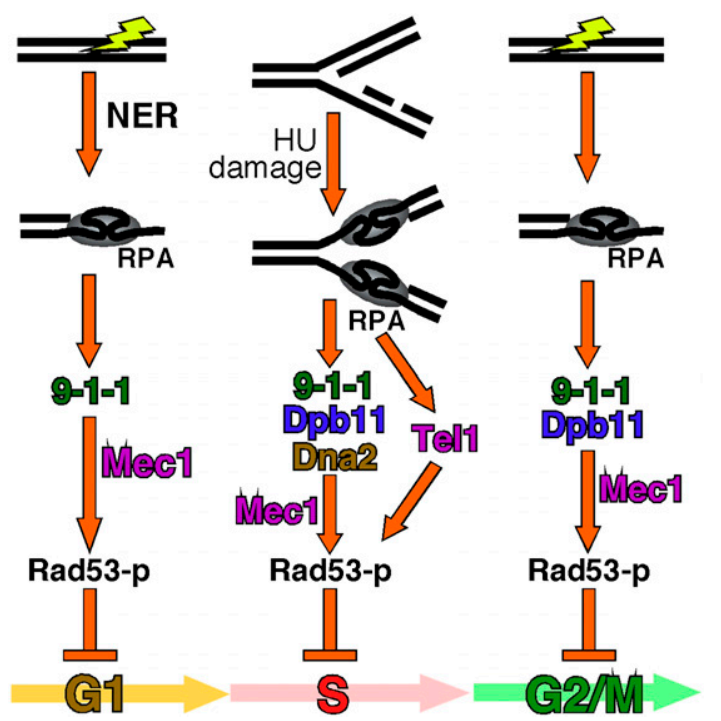

Figure 6. Model for Mecl activation during the cell cycle (see the text for details). kinase Rad53. Our analysis suggests that the hydroxyurea-induced replication checkpoint is predominantly transduced through Mrc1 phosphorylation (Fig. 3B; Supplemental Fig. 3A). The Mrc1 phosphorylation signal is significantly affected by mutation of TEL1 or DNA2 but less so by mutation of $D D C 1$. On the other hand, the S-phase DNA damage checkpoint is transduced through both Mrc1 and Rad9 phosphorylation. Phosphorylation of Rad9 in response to S-phase damage is attenuated most strongly by mutation of $D D C 1$, and that of Mrc1 is attenuated most strongly by mutation of both $D D C 1$ and TEL1 (Supplemental Fig. 3B). However, complete elimination of Rad53 phosphorylation in response to S-phase damage was only accomplished by mutation of all three genes: TEL1, DDC1, and DNA2. A tentative conclusion from these studies is that during $S$ phase, 9-1-1 signals primarily through the Rad9 mediator, whereas both 9-1-1 and Dna2 signal through Mrc1. Since these studies were carried out with a $d d c 1 \Delta$ strain and Dpb11's checkpoint function depend on its recruitment by Ddc1 (Navadgi-Patil and Burgers 2009), the individual checkpoint contributions of Ddc1 and Dpb11 could not be separated in this analysis.

Our data show an important and well-defined role for Dna2 in checkpoint initiation during S phase. Previous studies have shown a connection between Dna2 and the replication checkpoint. The growth defects of a tel1s mec1-21 strain, containing a hypomorphic allele of MEC1, was partially suppressed by overexpression of DNA2 (Vernon et al. 2008). In light of the demonstrated Mec1 stimulation function of Dna2, we propose that the mec1-21 allele is defective for kinase activation, and the increased abundance of Dna2 partially suppressed this defect.

The essential function of Dna2 is thought to stem from its role in Okazaki fragment maturation. During maturation, DNA polymerase $\delta$ carries out strand displacement synthesis that is coupled to concomitant cutting of the emerging flap by FEN1 nuclease and ligation by DNA ligase I (for review, see Burgers 2009). However, occasional excessive strand displacement synthesis generates long flaps that require cutting by Dna2. The essentiality of DNA2 is suppressed by mutations/conditions that disfavor strand displacement synthesis and therefore disfavor the generation of long flaps (Budd et al. 2006; Stith et al. 2008). Overexpression of RAD27, the gene encoding FEN1, suppresses the temperature sensitivity of several conditional mutants of DNA2, including that of

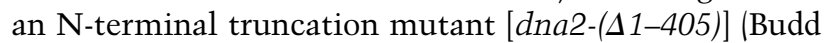
and Campbell 1997; Bae et al. 2001), indicating that this extensive truncation mutant showed Okazaki fragment maturation defects. However, both the dna2-WY-AA and dna2-( $\Delta 1-257)$ mutants showed only a detectable phenotype when combined with mutations in DDC1 and TEL1, and this growth phenotype was not suppressed by RAD27 overexpression (Supplemental Fig. 5A), suggesting that these latter mutants are not defective for Okazaki fragment maturation but for checkpoint function. DNA2 essentiality is also partially suppressed by deletion of RAD9 (Formosa and Nittis 1999; Budd et al. 2011), 
suggesting that lethality of $d n a 2 \Delta$ was the result of unrecoverable checkpoint arrest due to the generation of long ssDNA flaps. Surprisingly, however, we found that dna2s lethality was not suppressed in $d d c 1 \Delta$ mutants that are defective for the G1 and G2/M DNA damage

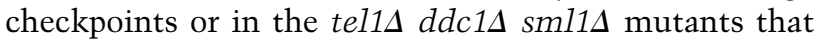
lost checkpoint activation in all phases of the cell cycle because of the inability to stimulate Mec1 kinase activity (Supplemental Fig. 4). Dna2s lethality was also not sup-

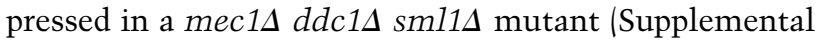
Fig. 5C). Therefore, we conclude that the suppression of dna2s lethality is $R A D$ 9-specific, and the connection to the DNA damage checkpoint may be more indirect.

Interestingly, both Dna2 and 9-1-1 are lagging strandspecific factors. Dna2 engages 5' flaps that are generated during the process of Okazaki fragment maturation, and 9-1-1 specifically loads onto $5^{\prime}$ double-stranded to singlestranded junctions, which occur naturally during Okazaki fragment priming (Ellison and Stillman 2003; Majka et al. 2006a). However, the possibility exists that checkpoint signaling on the leading strand could be possible after restart of a stalled replication fork by priming on the leading strand, which would provide the necessary 5' junction for 9-1-1 or Dna2 loading. Further studies to determine the role of 9-1-1 and Dna2 in replication restart and checkpoint activation may shed more light on checkpoint signaling initiated at the leading versus the lagging strand.

\section{Materials and methods}

All yeast strains and plasmids used in this study are listed in the Supplemental Tables. Cells were synchronized in G1 phase with $\alpha$ factor $(5 \mu \mathrm{g} / \mathrm{mL})$ and released into $S$ phase with or without hydroxyurea (100 or $200 \mathrm{mM}$ ) or 4-NQO $(2 \mu \mathrm{g} / \mathrm{mL})$; G2/M arrest was accomplished with nocodazole $(20 \mu \mathrm{g} / \mathrm{mL})$. Details are given in the Supplemental Material. Cell extracts were subjected to Western analysis with antibodies to Rad53, Mrc1, and Rad9 as described in the Supplemental Material.

\section{Phosphorylation assay}

The $20-\mu \mathrm{L}$ phosphorylation assay consisted of $25 \mathrm{mM}$ Hepes$\mathrm{NaOH}(\mathrm{pH} 7.8$ ), $5 \mathrm{mM} \mathrm{MgCl} 2,100 \mu \mathrm{M}$ unlabeled ATP, $0.5 \mu \mathrm{Ci}$ $\left[\gamma^{-}{ }^{32} \mathrm{P}\right]$ ATP, $1 \mathrm{mM}$ DTT, $100 \mathrm{mM} \mathrm{NaCl}, 100 \mu \mathrm{g} / \mathrm{mL}$ BSA, and 100 nM Rad53-kd with/without $2.5 \mathrm{nM}$ deca-primed single-stranded BlueScript DNA (3 kb), 150nM RPA, and 10nM Mec1/Ddc2. Reactions were initiated by adding the indicated amounts of (mutant) Dna2 and incubated at $30^{\circ} \mathrm{C}$. Reactions were terminated after $10 \mathrm{~min}$ by the addition of $5 \mu \mathrm{L}$ of $5 \times$ SDS-PAGE loading dye. Ten microliters of the samples was loaded onto $12 \%$ SDS-PAGE gel, dried, and exposed to a phosphor screen (GE Healthcare).

\section{Acknowledgments}

We thank Bonnie Yoder and Carrie Stith for strain construction and protein purification, Roberto Galletto for a gift of Pif1 and Rrm3 DNA helicases, Stephen Elledge for a gift of Mrc1 antibodies, Lynn White and Helen Piwnica-Worms for help with FACS analysis, and John Majors for critical discussions. This work was supported in part by Grants GM032431 and GM083970 from the National Institutes of Health.

\section{References}

Alcasabas AA, Osborn AJ, Bachant J, Hu F, Werler PJ, Bousset K, Furuya K, Diffley JF, Carr AM, Elledge SJ. 2001. Mrc1 transduces signals of DNA replication stress to activate Rad53. Nat Cell Biol 3: 958-965.

Ayyagari R, Gomes XV, Gordenin DA, Burgers PM. 2003. Okazaki fragment maturation in yeast. I. Distribution of functions between FEN1 and DNA2. J Biol Chem 278: 16181625.

Bae SH, Choi E, Lee KH, Park JS, Lee SH, Seo YS. 1998. Dna2 of Saccharomyces cerevisiae possesses a single-stranded DNAspecific endonuclease activity that is able to act on doublestranded DNA in the presence of ATP. I Biol Chem 273: 26880-26890.

Bae SH, Kim JA, Choi E, Lee KH, Kang HY, Kim HD, Kim JH, Bae KH, Cho Y, Park C, et al. 2001. Tripartite structure of Saccharomyces cerevisiae Dna2 helicase/endonuclease. Nucleic Acids Res 29: 3069-3079.

Bakkenist CJ, Kastan MB. 2003. DNA damage activates ATM through intermolecular autophosphorylation and dimer dissociation. Nature 421: 499-506.

Baldo V, Testoni V, Lucchini G, Longhese MP. 2008. Dominant TEL1-hy mutations compensate for Mec1 lack of functions in the DNA damage response. Mol Cell Biol 28: 358-375.

Bonilla CY, Melo JA, Toczyski DP. 2008. Colocalization of sensors is sufficient to activate the DNA damage checkpoint in the absence of damage. Mol Cell 30: 267-276.

Branzei D, Foiani M. 2009. The checkpoint response to replication stress. DNA Repair (Amst) 8: 1038-1046.

Budd ME, Campbell JL. 1997. A yeast replicative helicase, Dna2 helicase, interacts with yeast FEN-1 nuclease in carrying out its essential function. Mol Cell Biol 17: 2136-2142.

Budd ME, Choe W-C, Campbell J. 1995. DNA2 encodes a DNA helicase essential for replication of eukaryotic chromosomes. J Biol Chem 270: 26766-26769.

Budd ME, Reis CC, Smith S, Myung K, Campbell JL. 2006. Evidence suggesting that Pifl helicase functions in DNA replication with the Dna2 helicase/nuclease and DNA polymerase $\delta$. Mol Cell Biol 26: 2490-2500.

Budd ME, Antoshechkin IA, Reis C, Wold BJ, Campbell JL. 2011. Inviability of a DNA2 deletion mutant is due to the DNA damage checkpoint. Cell Cycle 10: 1690-1698.

Burgers PM. 2009. Polymerase dynamics at the eukaryotic DNA replication fork. I Biol Chem 284: 4041-4045.

Carle GF, Olson MV. 1985. An electrophoretic karyotype for yeast. Proc Natl Acad Sci 82: 3756-3760.

Chen X, Niu H, Chung WH, Zhu Z, Papusha A, Shim EY, Lee SE, Sung P, Ira G. 2011. Cell cycle regulation of DNA doublestrand break end resection by Cdk1-dependent Dna2 phosphorylation. Nat Struct Mol Biol 18: 1015-1019.

Choi JH, Lindsey-Boltz LA, Sancar A. 2007. Reconstitution of a human ATR-mediated checkpoint response to damaged DNA. Proc Natl Acad Sci 104: 13301-13306.

Delacroix S, Wagner JM, Kobayashi M, Yamamoto K, Karnitz LM. 2007. The Rad9-Hus1-Rad1 (9-1-1) clamp activates checkpoint signaling via TopBP1. Genes Dev 21: 14721477.

Duxin JP, Dao B, Martinsson P, Rajala N, Guittat L, Campbell JL, Spelbrink JN, Stewart SA. 2009. Human Dna2 is a nuclear and mitochondrial DNA maintenance protein. Mol Cell Biol 29: 4274-4282.

Ellison V, Stillman B. 2003. Biochemical characterization of DNA damage checkpoint complexes: Clamp loader and clamp complexes with specificity for $5^{\prime}$ recessed DNA. PLoS Biol 1: 231-243. 
Formosa T, Nittis T. 1999. Dna2 mutants reveal interactions with Dna polymerase $\alpha$ and $\mathrm{Ctf} 4$, a Pol $\alpha$ accessory factor, and show that full Dna2 helicase activity is not essential for growth. Genetics 151: 1459-1470.

Furuya K, Poitelea M, Guo L, Caspari T, Carr AM. 2004. Chk1 activation requires Rad9 S/TQ-site phosphorylation to promote association with C-terminal BRCT domains of Rad4TOPBP1. Genes Dev 18: 1154-1164.

Hartwell LH, Weinert TA. 1989. Checkpoints: Controls that ensure the order of cell cycle events. Science 246: 629-634.

Hennessy K, Lee A, Chen E, Botstein D. 1991. A group of interacting yeast DNA replication genes. Genes Dev 5: 958969.

Hu J, Sun L, Shen F, Chen Y, Hua Y, Liu Y, Zhang M, Hu Y, Wang $\mathrm{Q}, \mathrm{Xu} \mathrm{W}$, et al. 2012. The intra-S phase checkpoint targets dna2 to prevent stalled replication forks from reversing. Cell 149: 1221-1232.

Kondo T, Matsumoto K, Sugimoto K. 1999. Role of a complex containing Rad17, Mec3, and Ddc1 in the yeast DNA damage checkpoint pathway. Mol Cell Biol 19: 1136-1143.

Kumagai A, Lee J, Yoo HY, Dunphy WG. 2006. TopBP1 activates the ATR-ATRIP complex. Cell 124: 943-955.

Lee J, Kumagai A, Dunphy WG. 2007. The Rad9-Hus1-Rad1 checkpoint clamp regulates interaction of TopBP1 with ATR. J Biol Chem 282: 28036-28044.

Longhese MP, Paciotti V, Fraschini R, Zaccarini R, Plevani P, Lucchini G. 1997. The novel DNA damage checkpoint protein $\mathrm{ddc} 1 \mathrm{p}$ is phosphorylated periodically during the cell cycle and in response to DNA damage in budding yeast. EMBO I 16: 5216-5226.

Lundblad V, Szostak JW. 1989. A mutant with a defect in telomere elongation leads to senescence in yeast. Cell 57: 633-643.

Majka J, Binz SK, Wold MS, Burgers PM. 2006a. Replication protein A directs loading of the DNA damage checkpoint clamp to 5'-DNA junctions. I Biol Chem 281: 27855-27861.

Majka I, Niedziela-Majka A, Burgers PM. 2006b. The checkpoint clamp activates Mecl kinase during initiation of the DNA damage checkpoint. Mol Cell 24: 891-901.

Mallory JC, Petes TD. 2000. Protein kinase activity of Tellp and Meclp, two Saccharomyces cerevisiae proteins related to the human ATM protein kinase. Proc Natl Acad Sci 97: 13749-13754.

Mimitou EP, Symington LS. 2009. DNA end resection: Many nucleases make light work. DNA Repair (Amst) 8: 983995.

Mordes DA, Nam EA, Cortez D. 2008. Dpb11 activates the Mec1-Ddc2 complex. Proc Natl Acad Sci 105: 18730-18734.

Morrow DM, Tagle DA, Shiloh Y, Collins FS, Hieter P. 1995. TEL1, an S. cerevisiae homolog of the human gene mutated in ataxia telangiectasia, is functionally related to the yeast checkpoint gene MEC1. Cell 82: 831-840.

Myung K, Kolodner RD. 2002. Suppression of genome instability by redundant S-phase checkpoint pathways in Saccharomyces cerevisiae. Proc Natl Acad Sci 99: 4500-4507.

Navadgi-Patil VM, Burgers PM. 2009. The unstructured C-terminal tail of the 9-1-1 clamp subunit Ddc1 activates Mec1/ATR via two distinct mechanisms. Mol Cell 36: 743-753.

Navadgi-Patil VM, Kumar S, Burgers PM. 2011. The unstructured C-terminal tail of yeast Dpb11 (human TopBP1) protein is dispensable for DNA replication and the $S$ phase checkpoint but required for the G2/M checkpoint. I Biol Chem 286: 40999-41007.

Osborn AJ, Elledge SJ. 2003. Mrc1 is a replication fork component whose phosphorylation in response to DNA replication stress activates Rad53. Genes Dev 17: 1755-1767.
Parrilla-Castellar ER, Arlander SJ, Karnitz L. 2004. Dial 9-1-1 for DNA damage: The Rad9-Hus1-Rad1 (9-1-1) clamp complex. DNA Repair (Amst) 3: 1009-1014.

Pfander B, Diffley JF. 2011. Dpb11 coordinates Mecl kinase activation with cell cycle-regulated Rad9 recruitment. EMBO J 30: 4897-4907.

Puddu F, Granata M, Di Nola L, Balestrini A, Piergiovanni G, Lazzaro F, Giannattasio M, Plevani P, Muzi-Falconi M. 2008. Phosphorylation of the budding yeast 9-1-1 complex is required for Dpb11 function in the full activation of the UV-induced DNA damage checkpoint. Mol Cell Biol 28: 4782-4793.

Rouse J, Jackson SP. 2002. Interfaces between the detection, signaling, and repair of DNA damage. Science 297: 547-551.

Sanchez Y, Desany BA, Jones WJ, Liu Q, Wang B, Elledge SJ. 1996. Regulation of RAD53 by the ATM-like kinases MEC1 and TEL1 in yeast cell cycle checkpoint pathways. Science 271: 357-360.

Stith CM, Sterling J, Resnick MA, Gordenin DA, Burgers PM. 2008. Flexibility of eukaryotic Okazaki fragment maturation through regulated strand displacement synthesis. I Biol Chem 283: 34129-34140.

Vernon M, Lobachev K, Petes TD. 2008. High rates of 'unselected' aneuploidy and chromosome rearrangements in tell mecl haploid yeast strains. Genetics 179: 237-247.

Zhao X, Muller EG, Rothstein R. 1998. A suppressor of two essential checkpoint genes identifies a novel protein that negatively affects dNTP pools. Mol Cell 2: 329-340.

Zheng L, Zhou M, Guo Z, Lu H, Qian L, Dai H, Qiu J, Yakubovskaya E, Bogenhagen DF, Demple B, et al. 2008. Human DNA2 is a mitochondrial nuclease/helicase for efficient processing of DNA replication and repair intermediates. Mol Cell 32: 325-336.

Zhu Z, Chung WH, Shim EY, Lee SE, Ira G. 2008. Sgs1 helicase and two nucleases Dna2 and Exo1 resect DNA double-strand break ends. Cell 134: 981-994.

Zou L, Elledge SJ. 2003. Sensing DNA damage through ATRIP recognition of RPA-ssDNA complexes. Science 300: 15421548 . 


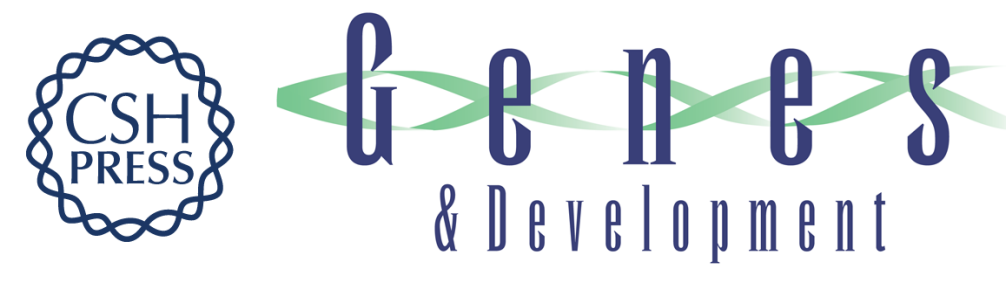

\title{
Lagging strand maturation factor Dna2 is a component of the replication checkpoint initiation machinery
}

\author{
Sandeep Kumar and Peter M. Burgers
}

Genes Dev. 2013, 27: originally published online January 25, 2013

Access the most recent version at doi:10.1101/gad.204750.112

\section{Supplemental http://genesdev.cshlp.org/content/suppl/2013/01/18/gad.204750.112.DC1 \\ Material}

Related Content $\quad \begin{aligned} & \text { Four pillars of the S-phase checkpoint } \\ & \text { Lee Zou }\end{aligned}$

Genes Dev. February , 2013 27: 227-233

References This article cites 50 articles, 28 of which can be accessed free at:

http://genesdev.cshlp.org/content/27/3/313.full.html\#ref-list-1

Articles cited in:

http://genesdev.cshlp.org/content/27/3/313.full.html\#related-urls

\section{License}

Email Alerting Receive free email alerts when new articles cite this article - sign up in the box at the top Service right corner of the article or click here.

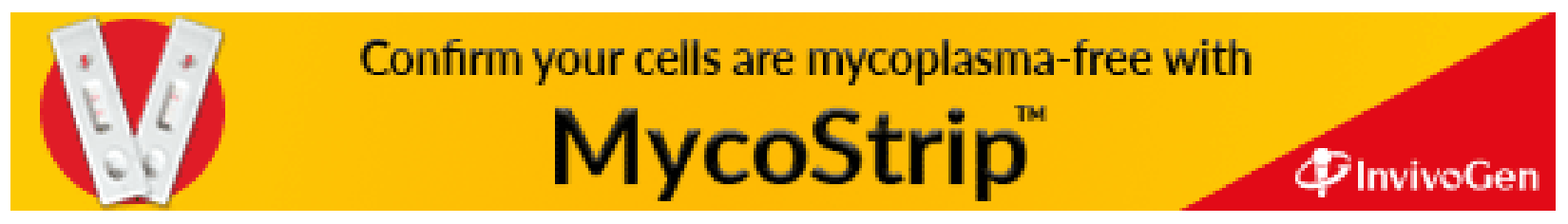

